\title{
$2^{\text {DITORIAL }}$
}

\section{Alan Saville \\ National Museums Scotland, UK}

Back to normal! As from this issue the EJA returns to its usual thrice-yearly format, but with the important difference that it should arrive in the hands of EAA members in the same month as specified on the cover.

The four articles herein admirably encompass a wide diversity of topics, approaches and interests, which is precisely what the EJA seeks to embrace in reflecting contemporary archaeological practice. Probing the potential of applying isotopic analysis to questions of diet and mobility in the past has been a regular feature of science-based articles in the EJA. In their contribution, Elisabeth Smits and colleagues continue with this theme and raise interesting new possibilities for the future utility of alternative stable isotopes which have not so far featured prominently in such studies, but which could become useful tools in Europe-wide investigations. Key to this work is scientific precision and quantification, meshed with the archaeological perspectives to frame the research and interpret the results, in this case relating to Neolithic settlement in the Lower Rhine Basin.

Martin Pitts is also concerned with quantification, using statistical analyses of assemblages of brooches and pottery to investigate the nature, relations and sociopolitical significance of the larger settlements of late Iron Age and early Roman Britain which are known collectively as oppida. This is a revisionist study, which challenges some recent interpretations and touches on general questions of identity, contact, tribalism, kingship, and urbanization on the fringes of the Roman world. The article by Marjan Galestin is similarly revisionist on a closely related topic, looking - in the context of The Netherlands - at which interpretation of the variable presence of Roman artefacts beyond the imperial frontier should be seen as the most plausible. Again there is a focus on ceramics, taking a rigorous archaeological approach which ends by advocating the need for detailed cataloguing of Roman finds beyond the Limes throughout Europe to permit broader comparative studies.

European Journal of Archaeology Vol. 13(1): 3-4 Copyright @ 2010 SAGE Publications ISSN 1461-9571 DOI: 10.1177/1461957110362938 
Galestin's call to arms, in terms of the need for archaeologists to press on with (or to rediscover) the fundamental requirement for material culture to be documented, arguably finds an echo in Sven Grabow's critique of European cultural heritage policy, which uses the flagship Santiago de Compostela pilgrim route of the Council of Europe's Cultural Routes Project as a case study. Grabow sees the implementation of this route as deeply flawed, intellectually and ideologically, and reveals how what should have been a starting point for the project - the collation, analysis and integration of the actual archaeological, architectural and historical evidence for the cult of St. James - has apparently been sidelined in the rush to develop what was a misguided, albeit well-intentioned, concept. If so, in a flagship pan-European project, these flaws would be deeply regrettable, but Grabow's postscript suggests that lessons have been learnt and recent developments in this area are better informed and researched.

Are we perhaps seeing a new trend manifested here in these articles, insofar as they seem closer in spirit and content to some of the old 'New Archaeology', with its concerns for quantitative and qualitative analysis, than to the approaches of the more freely interpretative post-processual archaeology that followed? The EJA takes no sides on the issues involved and is not prescriptive in its content, other than in terms of the quality and appropriateness of the articles it publishes. Those we publish are a genuine cross-section of the articles submitted, so it will be interesting to see if this is indeed a trend which continues with future issues. 